\title{
ON RANDOM APPROXIMATIONS AND A RANDOM FIXED POINT THEOREM FOR SET VALUED MAPPINGS
}

\author{
V. M. SEHGAL AND S. P. SINGH
}

\begin{abstract}
We prove a random fixed point theorem in a Banach space for set valued mappings and then derive a corollary that yields a fixed point theorem of BharuchaReid and Mukherjea, as a special case.
\end{abstract}

In a recent paper [1], Bharucha-Reid and Mukherjea proved the following stochastic analogue of the well-known Schauder's fixed point theorem.

THEOREM 1. Let $S$ be a compact and convex subset of a Banach space $E$ and $T$ : $\Omega \times S \rightarrow S$ be a continuous random operator. Then $T$ admits a random fixed point.

In this paper, we shall show that Theorem 1 can be derived from a more general result. For detailed definitions and terminologies we refer to Bharucha-Reid [1] or a recent paper of Itoh [3]. Throughout, this paper, $(\Omega, \Sigma)$ is a measurable space with $\Sigma$ a sigma algebra of subsets of $\Omega$. The symbol $2^{E}$ denotes the class of nonempty subsets of a Banach space $E$. A mapping $F: \Omega \rightarrow 2^{E}$ is called measurable iff for each open set $G$ of $E$,

$$
F^{-1}(G)=\{\omega \in \Omega: F(\omega) \cap G \neq \phi\} \in \Sigma .
$$

It may be pointed out that if $F(\omega)$ is compact for each $\omega \in \Omega$, then $F$ is measurable iff $F^{-1}(C) \in \Sigma$ for each closed subset $C$ in $E$ (see Himmelberg [2] or Itoh [3]).

Let $S$ be a nonempty subset of $E$. Let $T: \Omega \times S \rightarrow 2^{E}$ be a mapping. $T$ is called

(a) a random operator iff for each fixed $x \in S$, the mapping $T(\cdot, x): \Omega \rightarrow 2^{E}$ is measurable,

(b) upper (lower) semicontinuous (u.s.c, 1.s.c) iff for each fixed $\omega \in \Omega, T(\omega, \cdot)$ : $S \rightarrow 2^{E}$ is u.s.c (1.s.c), that is $(T(\omega, \cdot))^{-1}(C)$ is closed (open) subset of $S$ for each closed (open) subset $C$ of $E$,

(c) continuous iff $T$ is both u.s.c and 1.s.c.

A single valued measurable mapping $\phi: \Omega \rightarrow E$ is a random fixed point of the random operator $T: \Omega \times S \rightarrow 2^{E}$ iff $\phi(\omega) \in T(\omega, \phi(\omega))$ for each $\omega \in \Omega$.

The following selection theorem due to Kuratowski and Ryll-Nardzewski [4] is used in the proof of our result.

Received by the editors May 18, 1984.

1980 Mathematics Subject Classification. Primary 47H10, Secondary 54H25.

Kel words and phrases. Random fixed point, random operator, set valued mappings. 
Proposition 1. Let $S$ be a separable closed subset of $a$ Banach space $E$ and let $F$ : $\Omega \rightarrow 2^{S}$ be a measurable function such that $F(\omega)$ is compact for each $\omega \in \Omega$. Then there exists a single valued measurable function $\phi: \Omega \rightarrow S$ with $\phi(\Omega) \in F(\omega)$ for each $\omega \in \Omega$.

For subsets $A$ and $B$ of a normed space, we shall write

$$
d(A, B)=\inf \{\|x-y\|: x \in A, y \in B\} .
$$

The following result is a special case of a well-known result of Reich [5, Lemma 1.6].

Proposition 2. Let $S$ be a compact and convex subset of a Banach space $E$ and $F$ : $S \rightarrow 2^{E}$ be a continuous multifunction such that $F(x)$ is compact and convex for each $x$ in $S$. Then there exists an $x \in S$ with $d(x, F x)=d(F x, S)$.

The main result of this paper is

THEOREM 2. Let $S$ be a compact and convex subset of $E$ and $T: \Omega \times S \rightarrow 2^{E}$ be a continuous random operator with compact and convex values. Then there exists a single valued measurable map $\phi: \Omega \rightarrow S$ satisfying

$$
d(T(\omega, \phi(\omega)), \phi(\omega))=d(T(\omega, \phi(\omega)), S),
$$

for each $\omega \in \Omega$.

We first prove a few lemmas simplifying the proof of Theorem 2.

LEMMA 1. Let $S$ be a nonempty subset of a normed space $E$ and $T: S \rightarrow 2^{E}$ be a l.s.c. multifunction. If a sequence $\left\{x_{n}\right\}$ in $S$ converges to an $x_{0}$ in $S$, then for any $y_{0} \in T x_{0}$, there exists a subsequence $\left\{x_{n_{i}}\right\}$ of the sequence $\left\{x_{n}\right\}$ and a sequence $\left\{y_{n_{i}}\right\}$ with $y_{n_{i}} \in T\left(x_{n_{i}}\right)$ such that $y_{n_{i}} \rightarrow y_{0}$.

Proof. Let for each positive integer $n, N\left(y_{0}, 1 / n\right)$ be a neighborhood of $y_{0}$ of radius $1 / n$. Then $y_{0} \in N\left(y_{0}, 1 / n\right) \cap T x_{0}$. Consequently, for each $n$, there exists an $\varepsilon_{n}>0$ such that $T z \cap N\left(y_{0}, 1 / n\right) \neq \varnothing$ for each $z \in N\left(x_{0}, \varepsilon_{n}\right) \cap S$. Since $x_{n} \rightarrow x_{0}$, it follows that for each positive integer $k$, there exists $x_{n_{k}}, n_{k}>n_{k-1}$, such that $T x_{n_{k}} \cap N\left(y_{0}, 1 / k\right) \neq \varnothing$. If $y_{n_{k}} \in T x_{n_{k}} \cap N\left(y_{0}, 1 / k\right)$, then $\left\{y_{n_{k}}\right\}$ satisfies the conclusions of Lemma 1.

LEMMA 2. Let $S$ be a compact subset of a normed space $E$ and $T: S \rightarrow 2^{E}$ be a continuous multifunction with compact values. Then the real valued functions $f(x)=$ $d(x, T x)$ and $g(x)=d(T x, S)$ are continuous on $S$.

Proof. We show that $f$ is continuous. The proof that $g$ is continuous is similar.

Suppose $f$ is not continuous at some point $x_{0} \in S$. This implies the existence of an $\varepsilon>0$ and a subsequence $\left\{x_{n}\right\}$ in $S$ with $x_{n} \rightarrow x_{0}$ but

$$
\left|f\left(x_{n}\right)-f\left(x_{0}\right)\right|>\varepsilon
$$

for each $n$. Choose a $y_{0} \in T x_{0}$ such that $\left\|y_{0}-x_{0}\right\|=d\left(T x_{0}, x_{0}\right)$. By Lemma 1 , there exists a sequence $y_{n_{1}} \in T x_{n_{1}}$ with $y_{n} \rightarrow y_{0}$. Thus,

$$
f\left(x_{n_{1}}\right)=d\left(x_{n_{1}}, T x_{n_{1}}\right) \leqslant\left\|x_{n_{1}}-y_{n_{1}}\right\| \leqslant\left\|x_{n_{1}}-x_{0}\right\|+f\left(x_{0}\right)+\left\|y_{0}-y_{n_{i}}\right\| .
$$


This implies that $\lim \left(f\left(x_{n_{i}}\right)-f\left(x_{0}\right)\right) \leqslant \varepsilon$. Without loss of generality, we may assume that $\lim \left(f\left(x_{n}\right)-f\left(x_{0}\right)\right) \leqslant \varepsilon$. Now, for each $n$, choose a $y_{n} \in T x_{n}$ such that $d\left(x_{n}, T x_{n}\right)=\left\|x_{n}-y_{n}\right\|$. Since $T$ is L.s.c, $T S$ is compact and hence $\left\{y_{n}\right\}$ has a subsequence $\left\{y_{n_{i}}\right\} \rightarrow y_{1} \in T x_{0}$ for some $y_{1}$. Consequently,

$$
f\left(x_{0}\right)=d\left(x_{0}, T x_{0}\right) \leqslant\left\|x_{0}-y_{1}\right\| \leqslant\left\|x_{0}-x_{n_{i}}\right\|+f\left(x_{n_{i}}\right)+\left\|y_{n_{i}}-y_{1}\right\| .
$$

This yields $\lim \left(f\left(x_{0}\right)-f\left(x_{n_{i}}\right)\right) \leqslant \varepsilon$. Thus $\left|f\left(x_{n_{i}}\right)-f\left(x_{0}\right)\right| \leqslant \varepsilon$ eventually. This contradicts (1). Hence $f$ is continuous.

LEMMA 3. Let $S$ be a nonempty compact subset of a normed vector space $E$ and $T$ : $\Omega \times S \rightarrow 2^{E}$ be a multivalued random operator. Then, for each fixed $x \in S$, the mappings $g_{x}$ and $h_{x}$ defined by

$$
g_{x}(\omega)=d(T(\omega, x), x) \text { and } h_{x}(\omega)=d(T(\omega, x), S)
$$

are measurable.

Proof. Let $\alpha$ be a real. Then it is easy to verify that

$$
\left\{\omega \in \Omega: g_{x}(\omega)<\alpha\right\}=\{\omega \in \Omega: T(\omega, x) \cap N(x, \alpha) \neq \varnothing\} .
$$

This implies that $g_{x}$ is measurable. To show that $h_{x}$ is measurable, let $D$ be a countable dense subset of $S$. Then

$$
\begin{aligned}
\left\{\omega: h_{x}(\omega)<\alpha\right\} & \equiv \bigcup_{y \in D}\{\omega: d(T(\omega, x), y)<\alpha\} \\
& =\bigcup_{y \in D}\{\omega: T(\omega, x) \cap N(y, \alpha) \neq \varnothing\} .
\end{aligned}
$$

This implies that $h_{x}$ is measurable.

Proof of Theorem 2. Define a mapping $F: \Omega \rightarrow 2^{S}$ by

$$
F(\omega)=\{x \in S: d(T(\omega, x), x)=d(T(\omega, x), S)\} .
$$

Then it follows by Proposition 2 that $F(\omega) \neq \varnothing$. Further by Lemma 2, $F(\omega)$ is closed and hence a compact subset of $S$ for each $\omega \in \Omega$. We show that $F$ is measurable. Let $C$ be a closed subset of $S$ and $D$ a countable dense subset of $S$. For each $n$, let $D_{n}=\{x \in D: d(x, C)<1 / n\}$ and

$$
C_{n}=\bigcup_{x \in D_{n}}\left\{\omega \in \Omega: d(T(\omega, x), x)<d(T(\omega, x), S)+\frac{1}{n}\right\} .
$$

By Lemma 3, $C_{n}$ is measurable for each $n$. We show that $F^{-1}(C)=\bigcap_{n=1}^{\infty} C_{n}$. If $\omega \in F^{-1}(C)$, then $F(\omega) \cap C \neq \varnothing$. This implies that there exists an $x_{0} \in C$ with $d\left(T\left(\omega, x_{0}\right), x_{0}\right)=d\left(T\left(\omega, x_{0}\right), S\right)$. Since $D$ is dense in $S$, it follows by Lemma 2 , that for each fixed $n$, there exists an $x_{n} \in D$ such that $d\left(x_{n}, C\right)<1 / n$ and

$$
\begin{aligned}
d\left(T\left(\omega, x_{n}\right), x_{n}\right) & \leqslant d\left(T\left(\omega, x_{0}\right), x_{0}\right)+\frac{1}{2 n}=d\left(T\left(\omega, x_{0}\right), S\right)+\frac{1}{2 n} \\
& \leqslant d\left(T\left(\omega, x_{n}\right), S\right)+\frac{1}{n} .
\end{aligned}
$$


Thus $\omega \in \bigcap_{n=1}^{\infty} C_{n}$. Conversely, if $\omega \in \bigcap_{n=1}^{\infty} C_{n}$, then for each $n$, there exists an $x_{n} \in D_{n}$ with $d\left(T\left(\omega, x_{n}\right), x_{n}\right)<d\left(T\left(\omega, x_{n}\right)\right)+1 / n$. Since $\left\{x_{n}\right\} \subseteq S$ and $S$ is compact, there exists a subsequence $x_{n_{i}} \rightarrow x_{0} \in C$. This implies that $d\left(T\left(\omega, x_{0}\right), x_{0}\right)=$ $d\left(T\left(\omega, x_{0}\right), S\right)$. Thus $x_{0} \in F(\omega) \cap C$, that is, $\omega \in F^{-1}(C)$. This proves that $F$ is measurable. Consequently, by Proposition 1 there exists a single valued measurable function $\phi: \quad \Omega \rightarrow S$ with $\phi(\omega) \in F(\omega)$ for each $\omega \in \Omega$. This yields $d(T(\omega, \phi(\omega)), \phi(\omega))=d(T(\omega, \phi(\omega)), S)$.

The following, a special case of the above result, contains Theorem 1.

COROllary 1. Under the hypothesis of Theorem 2, if in addition $T(\omega, x) \subseteq S$ for each $\omega \in \Omega, x \in \partial S$ (boundary of $S$ ), then $\phi$ therein in Theorem 2, is a random fixed point of $T$.

Proof. If for some $\omega \in \Omega, T(\omega, \phi(\omega)) \cap S=\phi$, then $\phi(\omega) \notin \partial S$. Since $\phi(\omega) \in S$, it follows that $\phi(\omega)$ is an interior point of $S$. Choose a $y \notin T(\omega, \phi(\omega))$ such that $\|y-\phi(\omega)\|=d(T(\omega, \phi(\omega)), \phi(\omega))$. Since $y \notin S$, there exist a $\lambda, 0<\lambda<1$ such that $(1-\lambda) y+\lambda \phi(\omega) \in S$. This implies that

$$
\begin{aligned}
d(T(\omega, \phi(\omega)), S) & \leqslant\|y-((1-\lambda) y+\lambda \phi(\omega))\| \\
& =\lambda\|y-\phi(\omega)\|<d(T(\omega, \phi(\omega)), S) .
\end{aligned}
$$

This is impossible. Thus, for each $\omega \in \Omega, T(\omega, \phi(\omega)) \cap S \neq \varnothing$. This implies $d(T(\omega, \phi(\omega)), S)=0$ for each $\omega \in \Omega$. Hence by Theorem $2, d(T(\omega, \phi(\omega)), \phi(\omega))=$ 0 , that is, $\phi(\omega) \in T(\omega, \phi(\omega))$ for each $\omega \in \Omega$.

\section{REFERENCES}

1. A. T. Bharucha-Reid, Fixed point theorems in prohabilistic analysis, Bull. Amer. Math. Soc. 82 (1976), 641-657.

2. C. J. Himmelberg, Measurable relations, Fund. Math. 87 (1975), 53-72.

3. S. Itoh, Random fixed point theorems with an application to random differential equations in Banach spaces. J. Math. Anal. Appl. 67 (1979), 261-273.

4. K. Kuratowski and C. Ryll-Nardzewski, A general theorem on selectors, Bull. Acad. Polon. Sci. Sér. Sci. Math. Astronom. Phys. 13 (1965), 397-403.

5. Simeon Reich, Fixed poirts in locally convex spaces, Math. Z. 125 (1972), 17-31.

Di:PARTMENT OF MATHEMATICS, UNIVERSITY OF WyOMING, LARAMIE, WyOMING 82070

Dipartment of Mathematic's and Statistics, Memorial. University of Newfoundiand, St. JOHN`S. Ni:WhoUndi.AND, CANADA AlC 5S7 